http://dx.doi.org/10.18359/ravi.1496

\title{
Propuesta de diseño de un modelo educativo integral para capacitaciones corporativas $^{1}$
}

\author{
Edward Washington Rubio Guerrero ${ }^{2} *$ \& Marcela Georgina Gómez Zermeño $0^{3 * *}$ \\ Universidad Ricardo Palma, Perú* \\ Tecnológico de Monterrey, México**
}

Recibido, diciembre 15 de 2015

Concepto evaluación, febrero 02 de 2016

Aceptado, febrero 15 de 2016

\author{
Referencia: Rubio Guerrero, E.; Gómez Zermeño, M. \\ (2016). "Propuesta de diseño de un modelo educativo \\ integral para capacitaciones corporativas". Revista Academia \\ y Virtualidad, 9, (1), 52-67
}

\section{Resumen}

Este artículo plantea el diseño de una propuesta educativa para las capacitaciones corporativas desde la perspectiva de la universidad corporativa y empresas. El estudio se enfocó en el Centro de Educación Continua de una universidad corporativa donde se desarrollan programas de capacitación y educación virtual. La muestra se compuso por cuatro grupos: alumnos de programas ejecutivos, docentes de programas de capacitación, responsables del área de capacitación y recursos humanos, y un grupo de responsables de educación continua y virtual. La hipótesis del estudio especula que las capacitaciones corporativas que se diseñan sólo considerando los objetivos y necesidades de un solo grupo, no serán tan efectivas como las que toman en cuenta a los grupos involucrados. Se utilizó una metodología mixta para desarrollar un estudio sobre los procesos de formación corporativa que permita conocer cómo diseñar un modelo de capacitación corporativa acorde con las expectativas, objetivos y enfoques de los actores.

Palabras clave: capacitación corporativa, universidad corporativa, educación continua, entorno virtual de aprendizaje, modelo educativo.

\footnotetext{
1 Artículo de investigación científica y tecnológica como resultado del proyecto de investigación "Propuesta para el diseño de un modelo educativo integral para capacitaciones corporativas". Financiado por el Tecnológico de Monterrey.

2 Docente, Universidad Ricardo Palma, Santiago de Surco, Lima, Perú. Correo electrónico: edrubiog@gmail.com

3 Doctora, Tecnológico de Monterrey, Monterrey, Nuevo León. México. Correo electrónico: marcela.gomez@itesm.mx
} 


\title{
A design proposal of an integrated education model for corporate training
}

\begin{abstract}
This paper offers an education proposal design for corporate training from a perspective of a corporate university and business. The analysis was focused upon the Continuous Education Center of a corporate university where virtual training and education programs have been developed. The sample is composed by four groups: 1) students of executive programs; 2) teachers of training programs; 3) staff division of training and human resources; and 4) the staff group of permanent virtual education. Our research hypothesis points out that corporate training designed and based only on needs and goals of a single group are not as effective as those ones to consider all the involved groups. Then a mixed approach was used for analysis to discover a way to design a corporate training model according to the expectations, goals and focus of all the players.
\end{abstract}

Keywords: corporate training, corporate university, continuing education, virtual learning environment, education model.

\section{Proposta de desenho de um modelo educativo integral para capacitações corporativas}

\section{Resumo}

Este artigo planteia o desenho de uma proposta educativa para as capacitações corporativas desde a perspectiva da universidade corporativa e empresas. O estudo centrou-se no Cetro de Educação Continua de uma universidade corporativa, onde se desenvolvem programas de capacitação e educação virtual. A amostra ficou composta de quatro grupos: alunos de programas executivos, docentes de programas de capacitação, responsáveis da área de capacitação e recursos humanos, e um grupo de responsáveis de educação continua e virtual. A hipótese de estudo presume que as capacitações corporativas desenhadas somente considerando os objetivos e necessidades de um grupo só, não serão tão efetivas quanto as que tomam em conta aos grupos involucrados. Utilizou-se uma metodologia mista para desenvolver um estudo sobre processos de formação corporativa que permita conhecer como desenhar um modelo de capacitação corporativa acorde com as expetativas, objetivos e abordagens dos atores.

Palavras chave: capacitação corporativa, universidade corporativa, educação continua, entorno virtual de aprendizado, modelo educativo. 


\section{Introducción}

Las capacitaciones corporativas han constituido una alternativa de educación continua para los ejecutivos en la actualidad. Las empresas, preocupadas por impulsar este aspecto, han organizado sus áreas de capacitación y brindar espacios para el entrenamiento y formación con el objetivo de generar una mejor gestión, calidad y productividad.

En la nueva dinámica de la globalización, muchas empresas han adoptado el concepto de universidad corporativa utilizando recursos didácticos y modelos e-Learning para transmitir conocimientos y competencias requeridas por la empresa (Tamanini y Bergero, 2012). En el centro educativo en estudio, a través de sus áreas de educación continua y escuelas de negocios, se ha orientado la oferta educativa a un mayor público, buscando atender las necesidades de capacitación de las empresas. Mientras tanto, profesionales y ejecutivos también buscan mejorar su competitividad y obtener mejores oportunidades laborales.

Las tecnologías de la información y comunicación (TIC), también han influenciado en la gestión organizacional; por eso, los directivos vinculados a recursos humanos y a capacitación en empresas, tienen interés en la inclusión de TIC en los procesos de formación empresarial, las cuales se han convertido en mediadoras de los entornos de aprendizaje abiertos (Hannafin, Land y Oliver, 2002).

Para ello, entonces, se planteó el interrogante: ¿Cómo diseñar un modelo de capacitación corporativa orientado a satisfacer las necesidades y objetivos de todos los actores involucrados en este proceso? El objetivo principal es establecer los componentes y procesos en el diseño de un modelo de capacitación corporativa virtual.

En esa dirección, para orientar la investigación se planteó la siguiente hipótesis: las capacitaciones corporativas que se diseñan sólo tomando en consideración los objetivos y necesidades de un solo grupo, no serán tan efectivas como las que toman en cuenta a todos los grupos involucrados. Con esta investigación se espera contribuir al desarrollo de un modelo educativo que favorezca la gestión de la capacitación corporativa, reforzando su metodología aplicativa del aprendizaje.

\section{Marco teórico \\ Capacitaciones corporativas}

La capacitación está ligada directamente a los objetivos y necesidades de la organización, como afirma Blake (1997), quien sostiene que la capacitación busca satisfacer las necesidades que las organizaciones tienen de incorporar conocimientos, habilidades y actitudes en su personal, como parte de un proceso de cambio, crecimiento y adaptación a nuevas circunstancias internas y externas. Con la globalización, las empresas inician nuevas formas de gestión y vuelven su mirada al trabajador, a quien le otorgan un valor diferencial y se busca destacar su talento, su potencialidad para la empresa y reconocer sus habilidades y competencias.

La metodología de la capacitación corporativa es catalogada como práctica y aplicativa; son procesos educativos que responden a objetivos específicos de preparación y su aplicación es inmediata en el campo laboral. A diferencias de los modelos educativos de las universidades, éstos son modelos más flexibles, acordes con las necesidades reales de capacitación, para lo cual se usan diferentes recursos didácticos. Es importante medir el impacto de las capacitaciones. Al respecto, Gordillo (2004) habla de 4 niveles de evaluación. El nivel de reacción, vinculado a la satisfacción del proceso de capacitación que se aplica al participante; el nivel de conocimiento, que mide la adquisición de las competencias a través de un test de conocimientos; el tercer nivel de comportamiento y aplicabilidad, que mide las conductas adquiridas y las nuevas habilidades para el puesto de trabajo a través 
de la observación; por último, el cuarto nivel mide el retorno de inversión de la capacitación a través de los instrumentos económicos.

Una universidad corporativa es la transformación de un departamento de una empresa orientada a lograr un alineamiento efectivo entre la estrategia de desarrollo corporativo y la actividad formativa (Ferrer, 2009). Lorenzatti (2010), en su aporte, define la universidad corporativa como el proceso por medio del cual la organización integra el aprendizaje estratégico, permanente y de orientación hacia los resultados de la actividad, en toda su cadena de actividad productiva y fuerza laboral.

Respecto al concepto de universidad corporativa en Latinoamérica, un estudio realizado en 2011 por IE Business School y la Alianza Sumaq a más de 500 directivos de empresas de Latinoamérica, presenta cifras estadísticas sobre el crecimiento de esta corriente educativa en la región, destacando con un mayor número de universidades corporativas a Brasil (34\%), México (22\%), Argentina (14\%), Colombia (11\%) y Perú (10\%). Asimismo, resalta que $44 \%$ de las empresas top de Latinoamérica poseen una universidad corporativa (Cornejo, 2011).

En esa medida, se ha generado un gran debate entre el sector académico y empresarial respecto a la denominación y alcances de las universidades corporativas. En la tabla 1 se establece algunas diferencias entre la universidad tradicional y la universidad corporativa.

Tabla 1. Diferencias entre la universidad tradicional y universidad corporativa.

\begin{tabular}{|c|c|}
\hline $\begin{array}{l}\text { Universidad tradicional-Institución } \\
\text { académica }\end{array}$ & Universidad corporativa-Empresa \\
\hline $\begin{array}{l}\text { Se funda institucionalmente sobre principios } \\
\text { educativos y sociales para la formación del ser } \\
\text { humano }\end{array}$ & $\begin{array}{l}\text { Se funda sobre una estrategia corporativa de } \\
\text { formación del recurso humano }\end{array}$ \\
\hline $\begin{array}{l}\text { Se organiza mediante facultades y escuelas } \\
\text { académico profesionales que brindan carreras } \\
\text { universitarias }\end{array}$ & $\begin{array}{l}\text { Se organiza mediante centros de capacitación que } \\
\text { brinda programas de especialización y cursos }\end{array}$ \\
\hline Posee una planta de catedráticos por asignaturas & Posee una planta de capacitadores e instructores \\
\hline Promueve la investigación científica y académica & $\begin{array}{l}\text { Promueve la productividad y el desarrollo del } \\
\text { talento }\end{array}$ \\
\hline $\begin{array}{l}\text { Modelos educativos sustentados en modelos } \\
\text { pedagógicos de enseñanza-aprendizaje }\end{array}$ & $\begin{array}{l}\text { Modelos educativos flexibles y adaptados a } \\
\text { necesidades de capacitación }\end{array}$ \\
\hline Acreditación con certificación y títulos académicos & Entrega certificados de la empresa \\
\hline $\begin{array}{l}\text { Su público objetivo lo constituyen los alumnos de } \\
\text { pregrado, posgrado y de los programas de extensión } \\
\text { y educación continua y virtual. }\end{array}$ & $\begin{array}{l}\text { Su público objetivo no solo lo constituyen sus } \\
\text { trabajadores y ejecutivos sino también sus clientes } \\
\text { y proveedores. }\end{array}$ \\
\hline $\begin{array}{l}\text { Usa la tecnología como soporte de los procesos } \\
\text { formativos y administrativos. }\end{array}$ & $\begin{array}{l}\text { Posee gran experiencia en el manejo de la } \\
\text { tecnología y las incorpora en sus capacitaciones }\end{array}$ \\
\hline $\begin{array}{l}\text { Implementa reformas curriculares y cambios de } \\
\text { plan de estudios }\end{array}$ & $\begin{array}{l}\text { Adapta sus procesos de formación a los cambios } \\
\text { del mercado }\end{array}$ \\
\hline $\begin{array}{l}\text { Administra sus recursos económicos orientados a la } \\
\text { visión y misión educativa }\end{array}$ & $\begin{array}{l}\text { Administra sus recursos económicos con un } \\
\text { enfoque empresarial }\end{array}$ \\
\hline
\end{tabular}




\section{La educación continúa}

La educación continua abre un espacio educativo para estudiantes con mayor madurez, mejor motivación, con ciertas capacidades comunes que permitan acceder a logros formativos difíciles de conseguir en pregrado. La educación continua dentro del modelo de la sociedad del conocimiento, tiene como uno de sus principales objetivos desarrollar y mejorar las competencias profesionales y personales del individuo para la promoción social y laboral (López-Barajas, 2009). Es el medio para lograr objetivos globalizadores mediante estrategias educativas tradicionales y no convencionales; dentro de esta última se puede mencionar la capacitación corporativa. Letelier (2001) describe el perfil de los estudiantes de educación continua como personas adultas que poseen experiencia en un campo laboral y han pasado por una experiencia formativa.

\section{Entornos virtuales de aprendizaje}

Los entornos virtuales de aprendizaje constituyen un espacio de comunicación educativa con múltiples recursos tecnológicos. Salinas (2003) propone que este modelo permita que los alumnos se conviertan en usuarios de la formación, con un rol protagónico, con énfasis en el aprendizaje. Para comprender cómo se generan y desarrollan los entornos virtuales de aprendizaje se deben abordar desde dos aspectos: el pedagógico, referido a los procesos de aprendizaje y sus componentes, y el tecnológico respecto a los espacios y medios que soportan estos procesos (Asinsten, 2010).

El Informe sobre Educación a Distancia de la Comisión Asesora de la CONEAU (2004) recomienda que el modelo de aprendizaje debe estar orientado a valorar los procesos de comunicación y retroalimentación alumno-docente, especialmente los trabajos colaborativos que permitan construir conocimiento. Es importante tener en cuenta que los entornos virtuales deben poseer un clima propicio para los actores educativos, como los que proponen López, Ledesma y Escalera (2001): confianza, interacción, accesibilidad y motivación.

Con relación a los procesos de capacitación, de acuerdo con Gómez-Zermeño y Alemán (2011), impulsar los procesos de innovación en las empresas implica la implementación de sistemas que promuevan y fomenten el cambio y rendimiento dentro de la organización. Por tanto, requerir nuevos métodos de enseñanza en la sociedad del conocimiento demanda la utilización de medios y herramientas innovadoras.

\section{Metodología}

Esta investigación se realizó en la Universidad Ricardo Palma y su centro de Educación Continua, en Lima, Perú. En dicho centro se desarrollan programas de capacitación corporativa y de educación virtual. El trabajo se realiza en consideración de 4 perspectivas o variables que forman parte del contexto de las capacitaciones corporativas: (1) la primera variable la conforman los alumnos participantes de los programas ejecutivos y corporativos de la Universidad Ricardo Palma en la modalidad presencial y virtual a través de su Centro de Educación Continua; (2) la segunda variable la constituyen los docentes que dictan en estos programas de capacitación en esta universidad y en otras instituciones del medio; (3) la tercera variable la conforma la empresa a través de sus responsables de las áreas de capacitación y recursos humanos, que son clientes de la Universidad Ricardo Palma y también de otras universidades de Lima; (4) la cuarta variable a estudiar la constituye la universidad a través de sus responsables de las áreas de educación continua y virtual de la Universidad Ricardo Palma.

Para efectos concretos de este ejercicio investigativo, se ha elegido un enfoque mixto, debido a que el método cuantitativo permitirá estudiar las variables de alumnos, docentes y empresas, mientras que el método cualitativo servirá para estudiar la participación de la 
universidad, mediante la observación directa, interpretación y valoración de la información de las experiencias de capacitación corporativas. Se trata entonces de una investigación transversal por desarrollarse en un tiempo determinado y correlacionalcausal, porque establecerá relaciones entre las variables de estudio. Se trabajó con 4 variables independientes conformadas por los actores principales del proceso de capacitación: la empresa, la universidad, sus docentes y los estudiantes, en tanto que la variable dependiente de cada uno de ellos es la capacitación.

\section{Contexto de estudio}

La Universidad Ricardo Palma se encuentra ubicada en la ciudad de Lima, capital del Perú; es una institución educativa creada en 1969. Actualmente cuenta con más de 12,000 alumnos, 1200 docentes, 8 facultades que albergan sus 18 carreras profesionales. En julio del año 2000 la Universidad crea su Oficina de Educación Continua como unidad dependiente del rectorado. Desde 2004, dicho centro presentó un crecimiento debido a los diplomados ofrecidos y extendió su oferta educativa a través de curso cortos, cursos corporativos, cursos virtuales, programa en provincias, videoconferencias, entre otros. Estos programas se caracterizan por contar con un componente práctico en sus cursos $\mathrm{y}$ en el desarrollo de proyectos aplicativos por parte de los participantes. Se incorporan asimismo medios y recursos tecnológicos en el desarrollo de las materias, además sus planes de estudio están enmarcados en un ámbito de formación global. Esta área está innovando continuamente en temas de capacitación y proyectos educativos como el proyecto de educación virtual. En este punto, es importante mencionar que la universidad cuenta con un data center con un grupo de servidores que permite atender los servicios intranet, aula virtual, matrícula por internet, correos electrónicos, telefonía IP, helpdesk, página web, entre otros servicios y aplicaciones. Asimismo, posee todo un sistema de red inalámbrica y biblioteca virtual. Entre sus objetivos, se encuentra proveer de tecnología, capacitación y asesoría especializada en la implementación y producción de programas virtuales.

\section{Muestra de estudio}

Para determinar la muestra, se consideraron los grupos vinculados directamente a las capacitaciones corporativas, mediante la siguiente fórmula para establecer la muestra de cada grupo

n' $=\mathrm{S} 2 / \mathrm{V} 2=$ Tamaño provisional de la muestra $\mathrm{n}=\mathrm{n} /(1+(\mathrm{n} / \mathrm{N}))$ Tamaño de la muestra

De esta manera se plantea como muestra de estudio a 20 docentes que participan en programas de capacitación corporativa, 50 de dichos programas y 10 empresas que desarrollan cursos en el Centro de Educación Continua de la universidad en estudio. Para el caso del grupo de docentes y el grupo de empresas, la muestra recoge casi $100 \%$ de la población seleccionada por tratarse de grupos reducidos. Sin embargo, para el caso referido al grupo de los alumnos de estos programas cuya población asciende a 200 , se ha considerado un cálculo sobre un $90 \%$ de confianza y hasta un $10 \%$ de error por ser un grupo conocido, con el cual el investigador trabaja en forma permanente. Tal como se puede observar se recogen un total de 80 participantes para la muestra de la presente investigación.

\section{Instrumentos de recolección de datos}

Los instrumentos se constituyen en recursos de los cuales se valen los investigadores para aproximarse a los fenómenos y extraer la información. En cada uno de ellos se distinguen dos aspectos: forma y contenido, la primera referida a la técnica de investigación y la segunda a los indicadores que se presentan en las preguntas (Bernardo y Calderero, 2000).

Luego, se plantea para esta investigación un instrumento de medición que proporcione 
respuestas concretas, objetivas y de fácil procesamiento. Para este caso se ha elegido, según Giroux y Tremblay (2004), el método del cuestionario, el cual se conformó por preguntas estructuradas según las variables del estudio y a la vez respondió a los indicadores y propósitos de investigación y se llevó a cabo de manera anónima. Se construyeron 3 diferentes instrumentos para cada grupo (alumno, docente, recursos humanos o directivos) y se realizaron de manera virtual mediante Google Drive. El cuestionario incluyó preguntas a las metodologías utilizadas para la capacitación y el rol que desempeña cada actor, desde la perspectiva de sus contrarios.

Con la finalidad de revisar, evaluar y determinar la confiabilidad y validez del instrumento (Hernández, Fernández y Baptista, 1998), se aplicó una prueba piloto a 6 personas con perfiles similares a los de los grupos por encuestar; resultando en 2 encuestas pilotos por cada grupo de estudio.

El estudio de campo se dividió tres etapas: prueba piloto, lo cual permitió validar el instrumento y corregir errores; etapa de trabajo central, la aplicación del instrumento a los diferentes grupos y la recolección de información institucional sobre los procesos de capacitación corporativa.

\section{Análisis de resultados}

En primer lugar se presenta el perfil de los responsables de recursos y capacitación de las empresas participantes. Se debe mencionar que $60 \%$ estaba entre el rango de los 40 a 50 años, $30 \%$ en el rango de 30 a 40 años, mientras que $10 \%$ contaba con más de 50 años. Las empresas a las que pertenecían eran del sector público como privado. El tipo de profesión que se destaca entre los encuestados son: licenciado en relaciones industriales, licenciado en trabajo social, ingeniero industrial, administrador, psicólogo.

En relación con el perfil de los docentes encuestados, $38 \%$ se desempeña tanto como catedrático, capacitador de empresas y conferencista; $21 \%$ resalta su participación como capacitadores de empresas. Los docentes manifiestan tener igual porcentaje de experiencia capacitando tanto a instituciones del sector público como privado. Las edades de los docentes encuestados en $40 \%$ poseen más de 50 años; $30 \%$ pertenece al rango de edad de 30 a 40 años y el otro $30 \%$, de 40 a 50 años. Se encuestó a un $70 \%$ de docentes varones y a un $30 \%$ de docentes mujeres.

En relación con el perfil de los alumnos, 38\% se encontraba entre los 30 a 40 años de edad, $23 \%$ era menor a 30 años, $21 \%$ estaba entre 40 y 50 años, y más de 50 años el $17 \%$. Se encuestó $64 \%$ de alumnas mujeres y $34 \%$ de alumnos hombres. Las profesiones que resaltan de este grupo son: abogados, administradores, historiador, sociólogo, comunicador, traductor, psicólogo, ingeniero de sistemas.

Con la finalidad de mostrar los hallazgos del estudio, se abordan siete ejes temáticos: a) actores de la capacitación; b) objetivos, competencias y perfiles; c) temáticas de las capacitaciones; d) metodología; c) público objetivo y expectativa de la capacitación; e) capacitaciones y uso de medios tecnológicos; f) relación universidad, empresa y servicios de capacitación.

\section{a) Actores y protagonistas de la capacitación}

A lo largo de este estudio se han identificado claramente cuatro actores dentro del proceso de capacitación corporativa; dichos actores se desenvuelven en tres ámbitos: el institucional, donde se hallan la empresa y la universidad, definiendo el tipo de capacitación en un plan organizado; el ámbito del aprendizaje, donde se encuentran los otros dos actores: el alumno y el docente, protagonizando el proceso pedagógico del aprendizaje y, finalmente, el ámbito de la aplicación que permite que el alumno incorpore lo aprendido en su empresa. Al respecto, Guiñazú (2004) afirma que en toda capacitación se presentan dos contextos: el del aprendizaje, donde se realizan las actividades 
de capacitación, y el contexto de aplicación, donde se utiliza lo aprendido.

Los ejecutivos de recursos humanos $\mathrm{y}$ capacitación de las empresas encuestadas, identifican como los principales actores del proceso de capacitación corporativa con un $70 \%$ la triada conformada por empresatrabajador (alumno)-institución educativa, y bastante alejado con un 30\% se identifican cuatro actores: empresa-trabajador-docenteinstitución educativa.

En este escenario donde normalmente las empresas poseen un rol protagónico en todas las etapas del proceso de capacitación corporativa desde su diseño, implementación y evaluación, pues el $90 \%$ de los docentes encuestados sostiene que la universidad siempre debería asumir un papel protagónico en las capacitaciones corporativas. Esto significa una mayor participación en la estructura de los contenidos y la metodología de los programas en el marco de los objetivos y necesidades de capacitación de las empresas.

Asimismo, un $78 \%$ de los alumnos encuestados,considera que la universidad debe ser líder en mayor medida en estos procesos de capacitación, consolidandose aún más el rol protagónico que debe tener la universidad en los tres ámbitos: institucional, pedagógico y de aplicación.

El reto de la universidad y la empresa como organizadores y responsables de estos procesos educativas debe ser, tal como plantea Lopéz-Barajas (2009), desarrollar programas innovadores en la educación permanente que vayan acorde con los cambios que experimenta la sociedad actual.

\section{b) Objetivos, competencias y perfiles de las capacitaciones}

Uno de los principales objetivos de capacitación para una empresa, es buscar una mayor productividad y rendimiento en su trabajador, que alcanza el $29 \%$, esto a su vez se deriva en un mayor crecimiento para la empresa. También alcanza un interesante $24 \%$ las opciones de todas las anteriores y otros propósitos, lo que nos indica que las empresas persiguen diversos objetivos en sus capacitaciones.

Los alumnos ratifican en sus respuestas que uno de los principales propósitos de las empresas al organizar las capacitaciones para sus trabajadores es buscar una mayor productividad de los trabajadores. Así lo muestra un $92 \%$ que responde que siempre y casi siempre las capacitaciones persiguen el propósito de la productividad.

Del mismo modo, un $92 \%$ de los alumnos encuestados piensa que siempre o casi siempre las capacitaciones están orientadas a modernizar y mejorar los diferentes procesos de gestión de las empresas. Desde esta perspectiva, Lorenzatti (2010) sostiene que el aprendizaje forma parte esencial de la organización, pues apunta hacia aquellos conocimientos, habilidades y actitudes fundamentales que permitirán que todo el personal contribuya al logro de los planes estratégicos institucionales. En tal sentido, el objetivo de la empresa moderna no es solo seleccionar y contratar personal talentoso sino mantenerlos preparados y convertirlos en un elemento diferenciador de la imagen y competitividad corporativa (Ferrer, 2009).

El 39\% de los ejecutivos de recursos humanos encuestados considera que el perfil de un ejecutivo altamente capacitado es aquel que logra combinación efectiva de sus experiencias y conocimientos de manera práctica, seguido de cerca con un $22 \%$ que considera que su perfil pasa por ser altamente productivo.

Uno de los propósitos principales en el momento de definir los perfiles de capacitación de los ejecutivos, según los docentes, es lograr desarrollar sus habilidades y competencias en su espacio laboral y profesional. Esto queda demostrado de una forma contundente en el $100 \%$ de respuestas de los docentes encuestado 
que eligen las opciones de siempre y casi siempre. Asimismo, el 92\% de los alumnos encuestados de este grupo manifiestan que siempre y casi siempre las capacitaciones contribuyen a impulsar el talento humano del trabajador de cara al mercado laboral. Al respecto, Enebral (2012) sostiene que el nuevo trabajador, según Peter Drucker, es responsable, competente, desempeña su papel con autonomía, mejora mediante el aprendizaje y desarrollo permanente de sus conocimientos, habilidades, facultades y actitudes.

\section{c) Temáticas de la capacitación}

El tema que siempre se considera de gran relevancia para las diferentes empresas en sus capacitaciones corporativas está vinculado a la gestión empresarial. Este estudio lo resalta con un $28 \%$, seguido por temas de recursos humanos y de tecnología, cada uno de ellos con un $17 \%$. Si se toma en cuenta que estos temas también forman parte de la gestión, estarían sumando un $62 \%$.

Los temas principales de las capacitaciones corporativas para los docentes son los referidos a gestión empresarial y tecnología, tal como se demuestra, donde el rango de siempre y casi siempre alcanza un $80 \%$, lo que quiere decir que dentro del contexto de estos dos grandes temas se desarrollan capacitaciones en diferentes especialidades.

De otra parte, para los docentes encuestados existen otras temáticas relevantes vinculadas no sólo a la gestión de una empresa sino al desarrollo mismo de las personas y que se abordan con frecuencia, como los temas de liderazgo, coaching, comunicación y equipos, que alcanzan siempre y casi siempre, según los docentes encuestados un $60 \%$ de las preferencias de las empresas en sus capacitaciones.

El $55 \%$ de los docentes opina que siempre y casi siempre se eligen de forma correcta los temas de capacitación; sin embargo, se debe resaltar también que un $40 \%$ de los encuestados manifiesta que sólo algunas veces eligen de forma adecuada el tema de capacitación las empresas. En todo caso se trata de una afirmación que los docentes ven con cierta reserva.

Un $72 \%$ de los alumnos encuestados admite que siempre y casi siempre las capacitaciones responden a las necesidades de las empresas. En ese sentido las empresas identifican adecuadamente sus necesides y objetivos de capacitación antes de realizarlos. Sin embargo, existe un $24 \%$ que considera que no siempre llegan a adecuarse a las necesidades de capacitación.

\section{d) Metodología de las capacitaciones}

La metodología en las capacitaciones corporativas debe considerar para el $20 \%$ de los responsables de capacitación encuestados, el estudio de casos exitosos de otras empresa, el otro $20 \%$ se inclina por las dinámicas de evaluación aplicativas, y el 13\% manifiesta que hay que profundizar en los casos de la misma empresa; sumando estos tres se estaría hablando de un $53 \%$ que se inclina porque este tipo de capacitaciones deben ser totalmente prácticas.

Para el 95\% de los docentes encuestados, la metodología más adecuada para este tipo de capacitaciones es siempre y casi siempre también al caracterizarse por ser prácticas y aplicativas. Es decir, estos programas buscan una metodología participativa, dinámica, de casuísticas, de trabajos colaborativo en equipo, que logren impactar en un corto plazo en los procesos de gestión de la empresa que sean de la competencia del capacitado. No obstante, el 66\% de los alumnos encuestados que reciben este tipo de capacitaciones afirma que la propuesta metodológica que se aplica en las capacitaciones corporativas efectivamente es de orden práctica y aplicativa. En tanto, un $28 \%$ manifiesta que sólo algunas veces se aplica este tipo de metodología.

Respecto la medición de la efectividad de la 
metodología en las capacitaciones, Gordillo (2004) plantea cuatro niveles de evaluación: el nivel de reacción, que mide la satisfacción del participante; el nivel de conocimiento, que mide la adquisición de las competencias; el nivel de aplicabilidad, que mide las competencias adquiridas en relación al puesto de trabajo; y el nivel que mide el retorno de inversión de la capacitación.

\section{e) Público objetivo y expectativas de las capacitaciones}

El principal público objetivo que busca capacitar la empresa son sus trabajadores con un $35 \%$, seguido de cerca con un $30 \%$ de los ejecutivos de mando medio y un $26 \%$ por los directivos, aunque aún se puede apreciar que en las capacitaciones persiste una pirámide que comienza con el trabajador y continúa con los jefes hasta llegar a los directivos, los porcentajes cercanos que existen entre las diferentes categorías demuestran también que muchas empresas priorizan las capacitaciones de los directivos o jefes como punto de partida.

Mientras las empresas buscan entre sus principales objetivos de capacitación incentivar la productividad, los trabajadores buscan ser más competitivos y con ello lograr mejores oportunidades, estas dos últimas opciones representan un 50\% de sus expectativas. En un tercer lugar recién consideran la opción de productividad con un $19 \%$.

Los docentes encuestados manifiestan que los alumnos participantes tienen en sus expectativas respecto a las capacitaciones corporativas dos aspectos muy claros, que les pueden servir para mejorar su perfil profesional y que les brinde la opción de ascender y tener nuevas y mejores oportunidades laborales. Los docentes encuestados manifiestan en un 95\% que siempre o casi siempre los alumnos tienen estas expectativas cuando participan en procesos de capacitación.

El 27.46\% del grupo de alumnos encuestados participa siempre y casi siempre en las capacitaciones que organiza su institución. Esto significa que lo hacen regularmente. Un $29.41 \%$ también manifiesta que participan en las capacitaciones sólo en algunas oportunidades y un $27.45 \%$ manifiesta que lo hace esporádicamente. Esto significa que más de un $80 \%$ participa en capacitaciones de sus empresas en forma regular o eventualmente.

Los docentes consideran que el $60 \%$ de los alumnos de estas capacitaciones están bastante involucrados y participan frecuentemente durante el desarrollo de las clases, lo que estaría bastante ligado al tipo de metodología que se usan en estos cursos. Un $60.42 \%$ de alumnos encuestados, manifiesta que siempre o casi siempre las capacitaciones corporativas en las que participa son adecuadas, frente a un $31.25 \%$ que aún no se sienten convencidos con las capacitaciones que reciben, esto podría referirse a que no cumplen sus expectativas.

El $65 \%$ de los docentes piensan que sólo algunas veces los participantes asisten en forma obligada a las capacitaciones que organizan sus empresas y un $25 \%$ opina que esto sucede muy rara vez, en tanto que un 5\% manifiesta que nunca sucede. Si sumamos estos tres porcentajes se alcanzará un 90\% que piensa que pocas veces o casi nunca son obligados los alumnos a participar.

Un 74\% de los alumnos considera siempre y casi siempre los expositores que desarrollan las capacitaciones corporativas tienen experiencia y son conocedores de los temas que dictan, frente a un $20 \%$ que considera que no todos los expositores elegidos son los más adecuados.

El 70\% de los docentes encuestados participa siempre o casi siempre de capacitaciones corporativas en calidad de docente, expositor y /o conferencista, si se suma a ello el $25 \%$ que lo hace eventualmente, se podría estar hablando de que casi un $95 \%$ se encuentra participando actualmente como docente en este tipo de programas. 


\section{f) Capacitaciones y uso de medios tecnológicos}

Las empresas consideran, que la tecnología y la aplicación de ésta en los procesos de capacitación son muy necesarias, sumando ambas opciones a la necesidad y a la utilidad, se alcanzará un $69 \%$, lo que reafirma la tendencia que tienen las empresas de considerar estos recursos en el desarrollo de sus programas.

Para las empresas el uso del recurso tecnológico está bastante ligado al equipamiento tecnológico de los ambientes de clases, como lo demuestra un $39 \%$ que eligieron esta opción; sin embargo, hay una tendencia muy clara en el crecimiento vinculado al uso de los nuevos recursos de aprendizaje por internet como el aula virtual con un $28 \%$, las redes sociales con un $11 \%$ y la videoconferencias con un 6\%. Estos nuevos medios suman un importante $45 \%$ de preferencias. En este tipo de entornos virtuales de aprendizaje soportado por los nuevos medios, los alumnos se convierten en protagonistas de un proceso de formación flexible, donde el énfasis, según Salines (2003), se encuentra en el aprendizaje más que en la enseñanza, se busca ejercer una nueva relación con el saber, a través de nuevas prácticas de aprendizaje, adaptables a situaciones educativas en permanente cambio.

El 90\% de los docentes encuestados considera que siempre y casi siempre deben utilizarse las tecnologías en los programas de capacitación corporativa, pues están convencidos de que estos tipos de recursos facilitarán los procesos de capacitación.

\section{g) Relación universidad, empresa y servicios de capacitación}

Las empresas desde el momento que organizan una capacitación buscan que las instituciones educativas a las que contratan, bien sea universidades, institutos o entidades consultoras, desarrollen una capacitación a la medida de sus necesidades. Esta opción representa el 38\%, seguido muy de cerca con el 19\%, la opción que considera que se cumplan con los objetivos de capacitación, que en realidad es parte complementaria de la anterior, de esta manera se estaría hablando de un $57 \%$.

En cuanto a la oferta educativa, las capacitaciones en la medida en que se desarrollan para las empresas, alcanzan un $28 \%$ y constituye conjuntamente con los programas de educación continua con el otro $28 \%$, las dos ofertas que más demandan las empresas de hoy, ambas opciones que suman el 56\%, están referidas al desarrollo de cursos, diplomados y especilizaciones. No muy lejos se encuentra la opción de los posgrados con el $22 \%$ que siempre constituye una importante opción para todo profesional.

Los centros de educación continua de las universidades son vistos con especial interés por parte de las empresas porque ofrecen programas de formación y perfeccionamiento para los ejecutivos y profesionales, acordes con las necesidades del mercado, que van desde cursos cortos, especializaciones, diplomados hasta maestrías, que también se ofrecen de forma corporativa.

El $100 \%$ de los docentes encuestados considera que siempre y casi siempre debe existir una sociedad o alianza estratégica entre la empresa y universidad, para el desarrollo de los diversos programas de capacitación. Las empresas consideran que la universidad es un imporante respaldo académico para sus programas de capacitación, en tanto que las universidades ven también con gran interés la valiosa experiencias prácticas que poseen las empresas y que se aplican en sus capacitaciones.

Sólo un $40 \%$ de docentes encuestados piensa que siempre y casi siempre las universidades sólo deben intervenir certicando los programas de capacitación que hacen las empresas. Sin embargo, el $55 \%$ que se encuentra en el rango de las opciones algunas veces a nunca, piensan que la universidad tiene que ir más 
alla de extender los diplomas, pues debería involucrarse más en el diseño y desarrollo mismo de la capacitaciones.

Por su parte, los alumnos encuestados también consideran con un $78 \%$ que siempre y casi siempre debe realizarse un trabajo conjunto o alianza estratégica entre la universidad y empresa para organizar las capacitaciones corporativas.

Asimismo, el 62\% de los alumnos manifiesta que siempre y casi siempre la unviersidad debería participar certificando los progamas de capacitación de las empresas, lo que demuestra que los trabajadores consideran que la universidad debe participar directa $o$ indirectamente en estos procesos de capacitación.

Finalmente, los directivos de recursos humanos tienen bastante claro que el concepto de universidad corporativa obedece a una estrategia corporativa de capacitación con un $58 \%$, seguido de un $25 \%$ que considera que es una nueva forma de organizar un área de capacitación.

\section{Propuesta de capacitación corporativa}

Una vez realizado el análisis con los diferentes actores, se enuncian a continuación una serie de procesos académicos y administrativos que deben considerarse para la capacitación corporativa:

\section{Convocatoria del servicio de} capacitación: el punto de partida para una capacitación corporativa dentro del sector público son los términos de referencia, el cual es un documento trabajado por la institución donde se plasman la características de la capacitación solicitada que incluye: objetivos, temario, metodología, requisitos administrativos y montos presupuestados. Este documento se envía a las entidades educativas que puedan ofrecer el servicio. De acuerdo con dicho documento, la universidad realiza su propuesta académica y económica. En esta modalidad se encuentran mayormente las capacitaciones corporativas por presupuestos y por necesidades de áreas específicas. Para la capacitación por objetivos corporativos, normalmente el TDR solo exige la parte económica o cotización pues la parte académica lo define la misma empresa.

2. Contratación del servicio: cuando la empresa elige la entidad capacitadora, emite una orden de servicio o documento que autoriza la contratación del servicio de capacitación y a partir de este documento la universidad programa el desarrollo del curso, previa la aprobación por Acuerdo de Consejo Universitario, posteriormente al servicio de capacitación la universidad hace las gestiones de cobranza.

3. Informe y certificación: luego de concluida la capacitación, la universidad a través de sus docentes presenta un informe académico del desarrollo del curso a la empresa donde se resalta el desempeño de los participantes, su notas, la aplicabilidad de los aprendizajes y, finalmente, se certifican a los alumnos que aprobaron el curso, asimismo se desarrolla una evaluación del servicio obteniendo así una importante retroalimentación con los participantes.

La figura 1 muestra los principales hallazgos de la investigación y considera los procesos, actores y componentes de estos programas, válido para una modalidad presencial $\mathrm{y}$ especialmente para modalidad virtual. Este modelo presenta cuatro actores en el escenario de la capacitación: la empresa, como actor corporativo de la capacitación; la universidad, como actor académico de la capacitación; el docente, como actor pedagógico de la capacitación; y el alumno, como actor beneficiario de la capacitación. El modelo propone hasta tres niveles de relación $\mathrm{y}$ comunicación entre los actores del proceso de capacitación 


\section{PROPUESTA DE MODELO EDUCATINO PARA CAPACTIACIONES CORPORATIVAS}

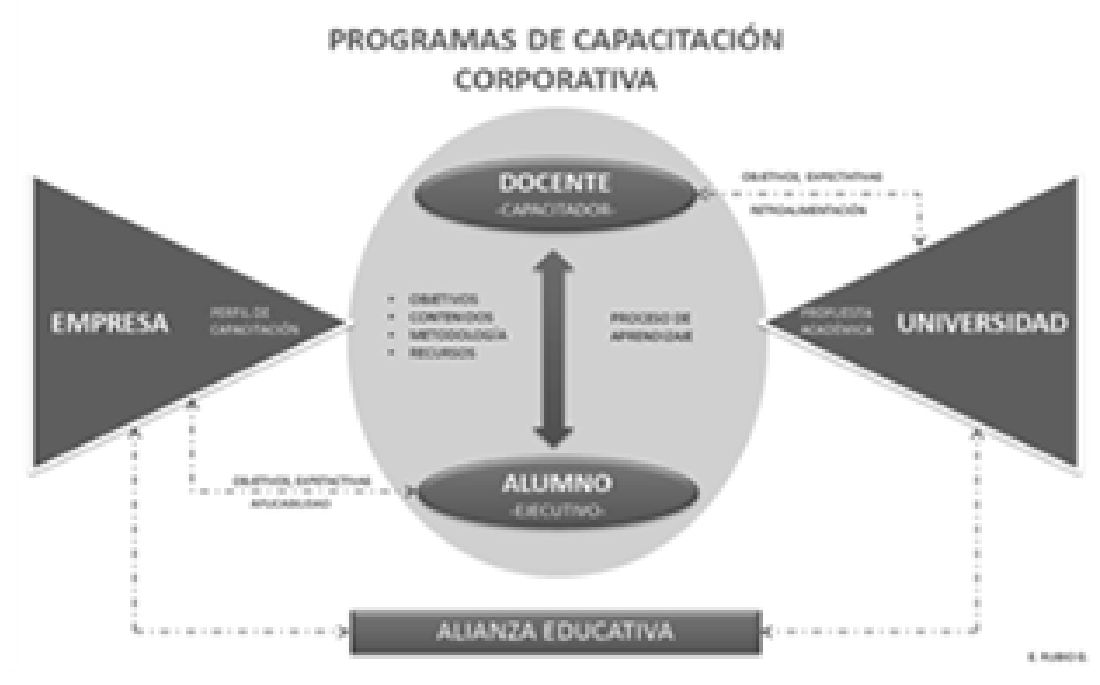

Figura 1. Diagrama de un modelo educativo para capacitaciones corporativas. Fuente: E. Rubio G.

1. El primer nivel es de tipo institucional. Si bien todo proceso de formación y aprendizaje tiene como núcleo central la relación docenteestudiante, en el caso de las capacitaciones corporativas hay una fuerte presencia institucional que impacta en este proceso; por un lado, se encuentran las empresas que requieren una capacitación en la medida en que lo plasman en su perfil del servicio de capacitación que desean contratar, que contienen los objetivos y necesidades de capacitación de la empresa, el público objetivo, las características metodológicas, perfil de expositores, cronogramas y presupuesto. Por otro lado, se encuentra la universidad que busca brindar servicio, y para ello prepara la propuesta académica que incluye objetivos, metodología, contenidos, expositores, evaluación, certificación, calendarioacadémico y la propuesta económica. Este tipo de relación institucional es importante enmarcarla dentro de una alianza educativa y estratégica, de tal manera que permita promover y facilitar los procesos de capacitación.
2. El segundo nivel de relación lo constituye el proceso mismo de aprendizaje que se desarrolla dentro de estas capacitaciones corporativas y cuyos actores lo constituyen el docente y el alumno participante de estas capacitaciones. En este proceso se deben tener en cuenta cuatro componentes que definen el perfil de la capacitación y que ha sido previamente estudiada y analizada institucionalmente por la empresa y la universidad, a saber:

- Objetivos: se establecen a la medida de las necesidades corporativas y pedagógicas.

- Contenidos: representan los temas seleccionados y estructurados de acuerdo con un plan de estudios.

- Metodología: la práctica en la realidad laboral y profesional de los participantes.

- Los recursos tecnológicos: brindarán un importante soporte a los procesos de capacitación.

3. El tercer nivel lo constituyen los procesos de retroalimentación que se desarrollan entre los diversos actores de la capacitación, que 
se encuentra muy ligados a las expectativas y propósitos de cada uno de ellos. Se debe destacar la retroalimentación empresa-alumno participante, donde se pretende medir el nivel de satisfacción alcanzado por ambos actores. La retroalimentación docente-universidad permite conocer y evaluar si los procesos de aprendizaje alcanzaron sus objetivos pedagógico, lo que permitirá mejorar las nuevas propuestas académicas de las capacitaciones corporativas.

\section{Conclusiones}

La pregunta de investigación planteó lo siguiente: ¿Cómo diseñar un modelo de capacitación corporativa orientado a satisfacer las necesidades y objetivos de todos los actores involucrados en este proceso? Así, se encontró que para llegar a diseñar este modelo se debe considerar: primero, que las empresas no busquen aprehender todos espacios que definen un proceso de capacitación, segundo, darle mayor protagonismo a las instituciones educativas, como especialistas, en el diseño de la propuesta pedagógica de los cursos, de tal manera que permita garantizar un proceso de aprendizaje dinámico e interactivo donde tanto el docente como el alumnos sean los verdaderos protagonistas.

En cuanto a la manera de integrar los diferentes objetivos y enfoques de capacitación de la empresa, el alumno y la universidad, se sugiere buscar un equilibrio en la participación de cada uno de estos actores, de este modo a la empresa le corresponde definir los objetivos corporativos y las necesidades específicas de capacitación del grupo y elección del tema.

Con esta información, la entidad educativa debe estudiar una propuesta académica adecuada que desarrolle un modelo pedagógico acorde con los programas solicitados por la empresa. El alumno, por su parte, debe asumir que estos programas permitirán el desarrollo de sus competencias y habilidades profesionales, de tal modo que haya motivación y se involucren activamente en los procesos de capacitación.
En relación con el componente virtual en las capacitaciones, se encontró que no se puede pretender diseñar un modelo exclusivo para la capacitación virtual corporativa sin antes comprender la problemática de los modelos presenciales.

El estudio identifica cuatro actores que intervienen en estos procesos de capacitación: la empresa, la entidad educativa, los docentes que imparten la capacitación y los alumnos. Los dos primeros actores establecen una relación de orden más administrativo e institucional más que académico para definir estos programas, en tanto el alumno y el docente se circunscriben en el proceso de aprendizaje. Las empresas siempre buscarán en las capacitaciones objetivos de productividad, en tanto los alumnos tengan expectativa en cuanto al desarrollo de sus competencias y cómo éstas permitan su crecimiento profesional.

Respecto a la hipótesis de investigación, la cual enuncia que las capacitaciones corporativas que se diseñan sólo tomando en consideración los objetivos y necesidades de un solo grupo no serán tan efectivas como las que toman en cuenta a todos los involucrados; se reveló que el protagonismo de las empresas en las capacitaciones corporativas se ve reforzado con la aparición de la universidad corporativa, donde las empresas manejan estratégicamente la organización de los programas de capacitación y convocan a la universidad como socio estratégico para respaldar académicamente sus capacitaciones, y dentro de este contexto, los alumnos y docentes se ven influenciados en los procesos de aprendizaje que desarrollan.

La propuesta del modelo pedagógico de estas capacitaciones debe equilibrar la participación de cada uno de los actores y esto es lo que exigen en las encuestas tanto los alumnos como los docentes de estas capacitaciones. 


\section{Referencias}

Asinsten, J. (2010). Producción de contenidos para la educación virtual. Guía de Trabajo del docente-contenidista. Virtual Educa Argentina- OEI. Documento recuperado de http://www.virtualeduca.org/documentos/ manual_del_contenidista.pdf.

Bernardo, J., \& Caldero, J. F. (2000). Investigación cuantitativa. Madrid: RIALP, SA.

Blake, O. (1997). La capacitación un recurso dinamizador de la organizaciones. Argentina Ediciones: Macchi.

Coneau (2004). Informe sobre educación a distancia. Comisión Asesora de la CONEAU. Buenos Aires 2004. Documento recuperado de http://www.coneau.gov.ar/archivos/477.pdf.

Cornejo, D. (2011). Universidades Corporativas: la sala de clases al interior de la empresa. Educamericas.com. Documento recuperado de http://www.educamericas. com/articulos/reportajes/universidadescorporativas-la-sala-de-clases-al-interior-dela-empresa.

Ferrer, E. (2009). Universidades Corporativas, políticas y alianzas estratégicas. En: Capital Humano, 232, 70-72. Documento recuperado de http://pdfs.wke.es/1/0/8/2/pd0000031082. pdf

Giroux, S. Y Tremblay, G. (2004). Metodología de las ciencias humanas. México D.F.: Fondo de Cultura Editorial.

Gómez-Zermeño, M. G., \& Alemán, L. Y. (2011). Administración de proyectos de capacitación basados en tecnología. Monterrey, Nuevo León, México: Editorial Digital del Tecnológico de Monterrey.

Gordillo, H. (2004). Las universidades corporativas, una nueva estrategia para la capacitación. Gestiopolis. Documento recuperado de http://www.gestiopolis.com/ canales $2 / \mathrm{rrhh} / 1 /$ unicorpo.htm

Guiñazú, G. (2004). Capacitación efectiva en la empresa. En: Invenio, 7(12), 103116. Documento recuperado de http://www. redalyc.org/pdf/877/87701209.pdf

Hannafin, M., Land, S., \& Oliver, K. (1999). Open learning environments: Foundations, methods, and models. En: Instructionaldesign theories and models: A new paradigm of instructional theory, 2, 115-140.

Hernández, S., Fernández, C. y Baptista, P. (2006). Metodología de la investigación. México D. F.: McGraw-Hill.

Letelier, M. (2001). Educación continua. Un desafío docente tridimensional. En; Revista Calidad en la Educación, 07. Documento recuperado de http://www.cned.cl/public/ secciones/seccionpublicaciones/doc/33/cse articulo100.pdf.

López-Barajas, E. (2009). El paradigma de la educación continua: reto del siglo XXI. Madrid, España: Narcea Ediciones/UNED.

López, A., Ledesma, R., \& Escalera, S. (2009). Ambientes Virtuales de Aprendizaje. México: Instituto Politécnico Nacional, Secretaría de Apoyo Académico. Documento recuperado de http://investigacion.ilce.edu.mx/panel_ control/doc/Rayon_Parra.pdf.

Lorenzatti M. (2010). La Universidad corporativa llegó para quedarse. En: America Learning and Media en Latinoamérica Edición, 006. Documento recuperado de http://www. americalearningmedia.com/edicion-006/72analisis/320-la-universidad-corporativa-llegopara-quedarse

Salinas, J. (2003). Entornos virtuales y formación flexible. Universidad de las Islas Baleares.Simposio Iberoamericano de virtualización del Aprendizaje y la Enseñanza. Documento recuperado de 
http:/gte.uib.es/pape/gte/publicaciones/ entornos-virtuales-y-formacion-flexible-0

Tamanini, E. \& Bergero H. (2012). Las universidades corporativas, un nuevo modelo de capacitación. En: Petrotecnia, 12. Documento recuperado de http://www. petrotecnia.com.ar/junio $12 /$ sinpublicidad/ Universidades.pdf. 